\title{
Dynamics and plasma properties of an X-ray jet from SUMER, EIS, XRT, and EUVI A \& B simultaneous observations *
}

\begin{abstract}
M. S. Madjarska
Armagh Observatory, College Hill, Armagh BT61 9DG, N. Ireland, UK

e-mail: madj@arm.ac.uk

Received 24 June 2010 / Accepted 27 October 2010

ABSTRACT

Context. Small-scale transient phenomena in the quiet Sun are believed to play an important role in coronal heating and solar wind generation. One of them, called "X-ray jet", is the subject of our study.

Aims. We intend to investigate the dynamics, evolution, and physical properties of this phenomenon.

Methods. We combine multi-instrument observations obtained simultaneously with the SUMER spectrometer onboard SoHO, with EIS and XRT onboard Hinode, and with EUVI/SECCHI onboard the Ahead and Behind STEREO spacecrafts. We derive plasma parameters such as temperatures and densities as well as dynamics by using spectral lines formed in the temperature range from $10000 \mathrm{~K}$ to $12 \mathrm{MK}$. We also use an image difference technique to investigate the evolution of the complex structure of the studied phenomenon.

Results. With the available unique combination of data we were able to establish that the formation of a jet-like event is triggered by not one, but several energy depositions, which are most probably originating from magnetic reconnection. Each energy deposition is followed by the expulsion of pre-existing or newly reconnected loops and/or collimated flow along open magnetic field lines. We derived in great detail the dynamic process of X-ray jet formation and evolution. For the first time we also found spectroscopically a temperature of $12 \mathrm{MK}$ (Fe XXIII $263.76 \AA$ ) and density of $4 \times 10^{10} \mathrm{~cm}^{-3}$ in the quiet Sun, obtained from a pair of Fe XII lines with a maximum formation temperature of $1.3 \times 10^{6} \mathrm{~K}$, in an energy deposition region. We point out a problem concerning an uncertainty in using the SUMER Mg X $624.9 \AA$ line for coronal diagnostics. We clearly identified two types of up-flow: one collimated up-flow along open magnetic field lines and a plasma cloud formed from the expelled BP loops. We also report a cooler down-flow along closed magnetic field lines. A comparison is made with a model developed by Moreno-Insertis et al. (2008).
\end{abstract}

Key words. Sun: corona - Sun: transition region - line: profiles - methods: observational

\section{Introduction}

X-ray jets in the solar atmosphere were first seen in images from the Soft X-ray Telescope (Tsuneta et al. 1991, SXT) onboard the Yohkoh satellite. They appear as collimated flows originating from coronal bright points (BPs) or active regions and are always associated with transient brightenings called microflares. We will review here only the very recent studies on these phenomena. Shimojo et al. (2007, and the references therein) resolved the fine structure of a X-ray jet and reported that a loop appeared near the footpoint of the jet when a footpoint brightening was observed. They also found that the X-ray jet appears after the loop breaks. They observed thread-like structures along the jet. Culhane et al. (2007b) found from Extremeultraviolet Imaging Spectrometer (EIS) 40" slot observations of a polar coronal hole that jet temperatures range from $0.4 \mathrm{MK}$ to 5.0 MK. The jet velocities had values that are less than the escape solar velocity $\left(\approx 618 \mathrm{~km} \mathrm{~s}^{-1}\right)$. They interpreted the increase of the radiance in the cooler spectral lines as emitted from the falling back plasma of the cooling down jet. Nishizuka et al. (2008) reported on simultaneous cool emission $\left(\sim 10^{4} \mathrm{~K}\right)$ in Ca II H images from the Solar Optical Telescope (SOT) onboard Hinode and hot emission $\left(1.3 \times 10^{6} \mathrm{~K}\right)$ in Fe XII $195 \AA$ images from the Extreme-ultraviolet Imaging Telescope (EIT)

* Figures $10-16$ and movie are only available in electronic form at http://www . aanda.org onboard the Solar and Heliospheric Observatory (SoHO) as well as in X-ray Telescope (XRT) images (Al_poly filter with a temperature response with a maximum at $5 \times 10^{6} \mathrm{~K}$ ) from a "giant" jet. From their observations of a current-sheet-like structure seen in all three instruments, they concluded that magnetic reconnection is occurring in the transition region or upper chromosphere. Cirtain et al. (2007) found that X-ray jets have two distinct velocities: one near the Alfvén speed $\left(\sim 800 \mathrm{~km} \mathrm{~s}^{-1}\right)$ and another near the sound speed $\left(200 \mathrm{~km} \mathrm{~s}^{-1}\right)$. Patsourakos et al. (2008) discussed the first stereoscopic observations of polar coronal jets made by the Extreme-ultraviolet imagers (EUVI) of the SECCHI instruments onboard the twin STEREO spacecrafts. The authors depicted a helical structure of a jet, which showed signs of untwisting with the jet initially ascending slowly with $\approx 10-20 \mathrm{~km} \mathrm{~s}^{-1}$ and then suddenly accelerating to velocities higher than $300 \mathrm{~km} \mathrm{~s}^{-1}$. Helical structures in jets have also been reported by Shimojo et al. (1996), Jiang et al. (2007), and Nisticò et al. (2009). Kamio et al. (2010) studied the relation of so-called macrospicules in He II $304 \AA$ and an X-ray jet. The Doppler shifts of the jet and the inclination angle of the jet were "attributed to a rotating motion of the macrospicule rather than a radial flow or an expansion". The authors concluded that the macrospicule is driven by the unfolding motion of a twisted magnetic flux rope, while the associated X-ray jet is a radial outflow.

It is believed that the majority of the jet-like phenomena in the solar atmosphere are produced by magnetic reconnection. 


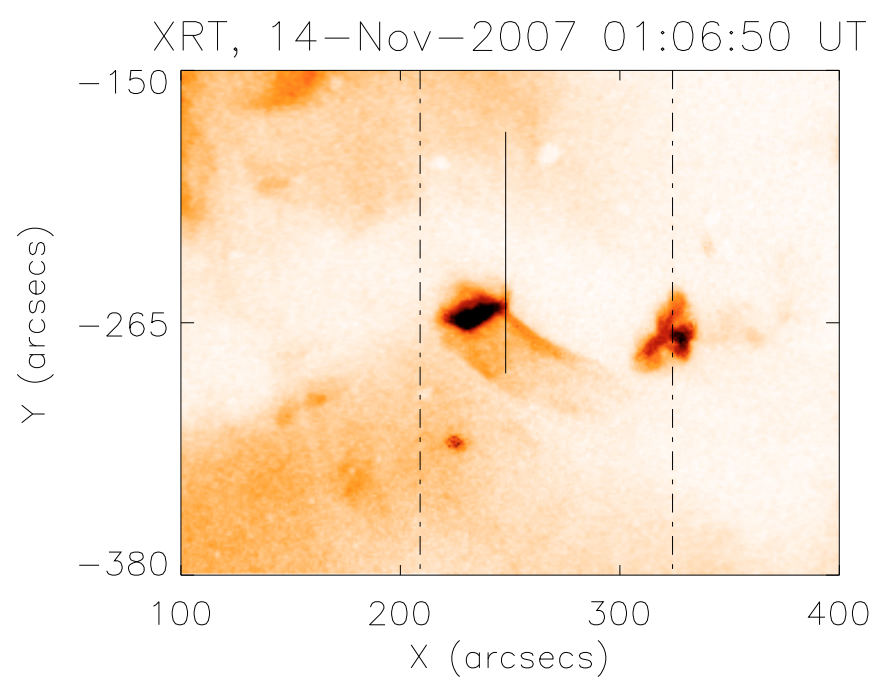

Fig. 1. Al_poly XRT image (colour table reversed) of the jet-like feature studied here. The vertical continuous line corresponds to the position of the SUMER slit. The dash-dotted line denotes the horizontal and part of the vertical field-of-view of the EIS raster.

Two-dimensional (Heyvaerts et al. 1977; Yokoyama \& Shibata 1995, 1996; Isobe et al. 2007), 2.5 dimensional (Karpen et al. 1998; Archontis et al. 2007) as well as three-dimensional (3D) (Moreno-Insertis et al. 2008; Pariat et al. 2009) magnetohydodynamic (MHD) models have been developed.

The feature analysed here was selected from a large number of jet-like events registered during a multi-instrument observing campaign. We exploit here the very rare circumstance that such a jet-like event was simultaneously registered by two spectrometers and several imagers. Because of the complexity of both the analysis and the amount of information contained in the data, we decided to present this study in an individual article, while a statistical study on the dynamics and physical parameters of jet-like events will follow in a separate publication. Section 2 contains detailed information on all analysed observations including the alignment, both spatially and temporally, of the different types of observations. Section 3 presents the analysis and gives the results on the dynamics, temperature, and density of the event. The obtained physical parameters and dynamics compared to already published similar information and a discussion on the observable characteristics of jet-like phenomenon in the light of a theoretical 3D MHD model by Moreno-Insertis et al. (2008) are given in Sect. 4.

\section{Observations}

The event discussed here occurred at the boundaries of an equatorial hole on 2007 November 14 (Fig. 1). It was registered with the Solar Ultraviolet Measurements of Emitted Radiation (SUMER) spectrometer onboard SoHO, EIS and XRT onboard Hinode, and with the EUVI in the Fe IX/X $171 \AA$ channel on board the A and B STEREO spacecrafts during a specially planned multi-instrument observing campaign. The fields-ofview (FOVs) of XRT, EIS, and SUMER are shown in Fig. 1. Details on the registered SUMER spectral lines can be found in Table 1. The analysed EIS spectral lines are listed in Table 2.
Table 1. SUMER spectral lines.

\begin{tabular}{ccc}
\hline \hline Ion & Wavelength $(\AA)$ & $\log T_{\max } / \mathrm{K}$ \\
\hline $\mathrm{N}$ V & 1238.82 & 5.3 \\
$\mathrm{C}$ I & 1248.00 & 4.0 \\
& 1248.88 & \\
$\mathrm{C}$ I & 1249.00 & 4.0 \\
$\mathrm{O}$ IV/2 & $1249.24 \mathrm{~b}$ & 5.2 \\
$\mathrm{Si} \mathrm{X} / 2$ & $1249.40 \mathrm{~b}$ & 6.1 \\
$\mathrm{C}$ I & 1249.41 & 4.0 \\
$\mathrm{Mg} \mathrm{X} / 2$ & 1249.90 & 6.1 \\
$\mathrm{Si}$ II & 1250.09 & 4.1 \\
$\mathrm{O}$ IV/2 & $1250.25 \mathrm{~b}$ & 5.2 \\
$\mathrm{Si}$ II & 1250.41 & 4.1 \\
$\mathrm{C}$ I & $1250.42 \mathrm{~b}$ & 4.0 \\
$\mathrm{~S} \mathrm{II}$ & 1250.58 & 4.2 \\
$\mathrm{Si}$ II & 1251.16 & 4.1 \\
$\mathrm{C}$ I & 1251.17 & 4.0 \\
$\mathrm{Si} \mathrm{I}$ & 1256.49 & 4.0 \\
$\mathrm{Si} \mathrm{I}$ & 1258.78 & 4.0 \\
$\mathrm{~S}$ II & $1259.53 \mathrm{~b}$ & 4.2 \\
$\mathrm{O}$ V 2 & 1259.54 & 5.4 \\
$\mathrm{Si}$ II & 1260.44 & 4.1 \\
\hline
\end{tabular}

Notes. The expression " $/ 2$ " means that the spectral line was observed in second order. The comment " $b$ " denotes that the spectral line is blending a close-by line.

Table 2. EIS observed spectral lines.

\begin{tabular}{ccc}
\hline \hline Ion & Wavelength $(\AA)$ & $\log T_{\max } / \mathrm{K}$ \\
\hline He I & $256.32 \mathrm{~b}$ & 4.7 \\
O V & 192.90 & 5.4 \\
O VI & 184.12 & 5.5 \\
Mg VI & $270.39 \mathrm{~b}$ & 5.7 \\
Mg VII & 278.39 & 5.8 \\
& 280.75 & 5.8 \\
Si VII & 275.35 & 5.8 \\
Fe VIII & 185.21 & 5.8 \\
Fe X & 184.54 & 6.0 \\
Fe XI & 188.23 & 6.1 \\
Si X & 258.37 & 6.1 \\
& 261.04 & 6.1 \\
Fe XII & 195.12 & 6.1 \\
Fe XIII & 202.04 & 6.2 \\
& 203.82 & 6.2 \\
Fe XIV & $274.20 \mathrm{~b}$ & 6.3 \\
Fe XV & 284.16 & 6.3 \\
Fe XVI & 262.98 & 6.4 \\
Ar XV & 293.69 & 6.6 \\
Fe XXIII & 263.76 & 7.1 \\
\hline
\end{tabular}

Notes. The comment " $b$ " indicates that the spectral line is blended (see Young et al. 2007; Brown et al. 2008; Young et al. 2009).

\subsection{SUMER}

The SUMER (Wilhelm et al. 1995; Lemaire et al. 1997) observations were made from 01:01 UT until 02:56 UT during a dedicated multi-instrument observing campaign targeting coronal hole boundaries. A slit with a size of $1^{\prime \prime} \times 300^{\prime \prime}$ was used. The detector B was exposed for $60 \mathrm{~s}$ while the spectrometer was observing in a sit-and-stare mode. The time sequence was followed by a raster with a size of $60^{\prime \prime} \times 300^{\prime \prime}$ made only in the O V $629.73 \AA$ and Si I $1256.49 \AA$ lines. The observations were compensated for the solar rotation. Five spectral windows were transferred to the ground, each with a size of 
50 spectral pixels $\times 300$ spatial pixels. The spectral line readouts are shown in Table 1. From all lines only O v $629.73 \AA$ was taken on the bare part of the detector. At the start of the observations (at 01:01 UT) the spectrometer was pointed at the solar disk coordinates $x$ cen $=-217^{\prime \prime}$ and ycen $=257^{\prime \prime}$.

\subsection{TRACE}

During the TRACE (Handy et al. 1999) observations on November 14, i.e. the period before a full eclipse, the effects of atmospheric absorption are already present (they occur for several weeks either side of the interval when TRACE is fully eclipsed and extends roughly from mid-October to the beginning of March). That is why we selected the three ultraviolet filters at $1550 \AA, 1600 \AA$, and $1700 \AA$, which are not influenced by the absorption of the Earth atmosphere during this period of time. Images in the Fe IX/X $171 \AA$ filter were taken with a cadence of $30 \mathrm{~min}$ and were used for co-alignment purposes. The observations started at 03:27 UT, i.e. $\approx 3 \mathrm{~h}$ later than SUMER and Hinode. The three UV channel observations were obtained with a cadence of $10 \mathrm{~s}$. The FOV of the images is $256^{\prime \prime} \times 256^{\prime \prime}$ with a spatial resolution of $1^{\prime \prime}$.

\subsection{EIS}

The EIS (Culhane et al. 2007a) study was specially designed to provide the best possible coverage of spectral lines with formation temperatures $\left(\log T_{\max }\right)$ from 4.7 to $7.1 \mathrm{~K}$ (Table 2). First, a raster with a size of $120^{\prime \prime} \times 512^{\prime \prime}$ was obtained followed by 50 rasters with a size of $24^{\prime \prime} \times 512^{\prime \prime}$ starting at 00:20 UT. The feature analysed in this work was registered only in the big raster covering the entire area of the jet from the start to the end of the eruption (Fig. 1). An exposure time of $60 \mathrm{~s}$ and $2^{\prime \prime}$ slit were used during these observations.

\subsection{MDI}

There exists one longitudinal magnetogram taken at 00:03:01 UT and one at 01:39:01 UT from the Michelson Doppler Imager (MDI, Scherrer et al. 1995) onboard SOHO as part of a 96-min cadence series, i.e. $50 \mathrm{~min}$ before the jet started and almost 15 min after the end of the event (Fig. 3). The magnetograms have an angular pixel size of $1.98^{\prime \prime}$, resulting in $\approx 4^{\prime \prime}$ spatial resolution and $1 \sigma$ noise of $20 \mathrm{G}$ (Scherrer et al. 1995). Unfortunately, the SOT/Hinode field-of-view ends just above the dynamic feature. We note here that we presently investigate several events (a few tens) taken with the same observing programmes, which were fully covered by SOT/Hinode at a cadence of $40 \mathrm{~s}$ so that the photospheric longitudinal magnetic field evolution can be investigated in relation to jet-like events.

\section{5. $X R T$}

The XRT (Golub et al. 2007) was observing with the Al_poly filter in a FOV of $384^{\prime \prime} \times 384^{\prime \prime}$. The exposure time was $16.38 \mathrm{~s}$ and the cadence $40 \mathrm{~s}$.

\subsection{EUVI/SECCHI}

EUVI/SECHHI data from both the Behind (B) and Ahead (A) spacecrafts of the STEREO mission were used in the present study taken between 00:20 UT and 2:00 UT. The cadence of
EUVI A was variable, but the exposure time was constantly $4 \mathrm{~s}$ with the exception of eight images (out of 82) where the exposure time was $16 \mathrm{~s}$. EUVI B recorded only 47 images in the above mentioned period with variable cadence and constant exposure time of approximately $4 \mathrm{~s}$ with one exception of $16 \mathrm{~s}$.

\subsection{Data reduction and co-alignment of the different instruments}

The data from all instruments were reduced using standard software. Only for SUMER the standard data reduction software could not provide a satisfactory level of data corrections for various instrumental effects. We applied additional corrections for the flatfield and geometrical distortion.

All images were de-rotated to a reference time of 02:27 UT, which was a good compromise between the data taken with the different instruments. The co-alignment of the instruments was based on aligning structures seen at similar temperatures. We used a TRACE 171 $\AA$ image taken at 03:27 UT and co-aligned it with an XRT image taken at 02:30 UT. The Al_poly filter has a peak response at $\log T=6.9 \mathrm{~K}$, but it can also register emission at temperatures as $\operatorname{low}$ as $\log T=5.5 \mathrm{~K}$. A few bright coronal structures, which showed little changes during $2 \mathrm{~h} 30 \mathrm{~m}$ of observations, were co-aligned. Next an XRT image of the peak of the jet evolution was aligned with the XRT image from 02:30 UT. Knowing the internal offsets of the TRACE channels, we selected a TRACE $1550 \AA$ image taken at the same time of the event as the TRACE $171 \AA$ image already aligned with XRT. Then the TRACE $1500 \AA$ was co-aligned with the SUMER O V $629.73 \AA$ and Si I $1256.49 \AA$ raster images. Finally, the EIS raster was co-aligned with all above mentioned instruments by using spectral lines with the best-suitable formation temperatures. The precision of the alignment is $1^{\prime \prime}-2^{\prime \prime}$. In Fig. 10 the images used for alignment are shown. Note that the different appearance of some structures is owing to the time difference of the obtained images. Nevertheless, there exist long-living structures that remain almost unchanged during the observations. The final adjustment was made from the comparison of the blue-shifted emission of EIS He II 256.32 A with the SUMER O V $629.73 \AA$ and N V $1238.82 \AA$. Because of the dynamic nature of the observed phenomenon, the synthesis of spectroscopic and imager data permitted us to easily combine the STEREO data with the rest of the observations. The MDI magnetograms were aligned with respect to TRACE $1550 \AA$ and SUMER Si I $1256.49 \AA$. The contours of the magnetic polarities are overplotted on the XRT and Si I $1256.49 \AA$ Amages in Fig. 10 .

\section{Data analysis and results}

The phenomenon presented here was detected in the course of a statistical study of brightening events in the quiet Sun and coronal holes (Subramanian et al. 2010). Subramanian et al. (2010) showed that numerous brightenings occur along coronal hole boundaries and inside coronal holes. Most of them were visually identified as jet-like features from pre-existing coronal bright points. Their spatial appearance suggested that they are a product of magnetic reconnection between the open magnetic field lines of coronal holes and the closed ones of pre-existing magnetic loops named coronal bright points.

The distinctiveness of our analysis lies in the use of the so far best combination of imager and spectroscopic data that were taken simultaneously and cover the entire lifetime of a jet-like 

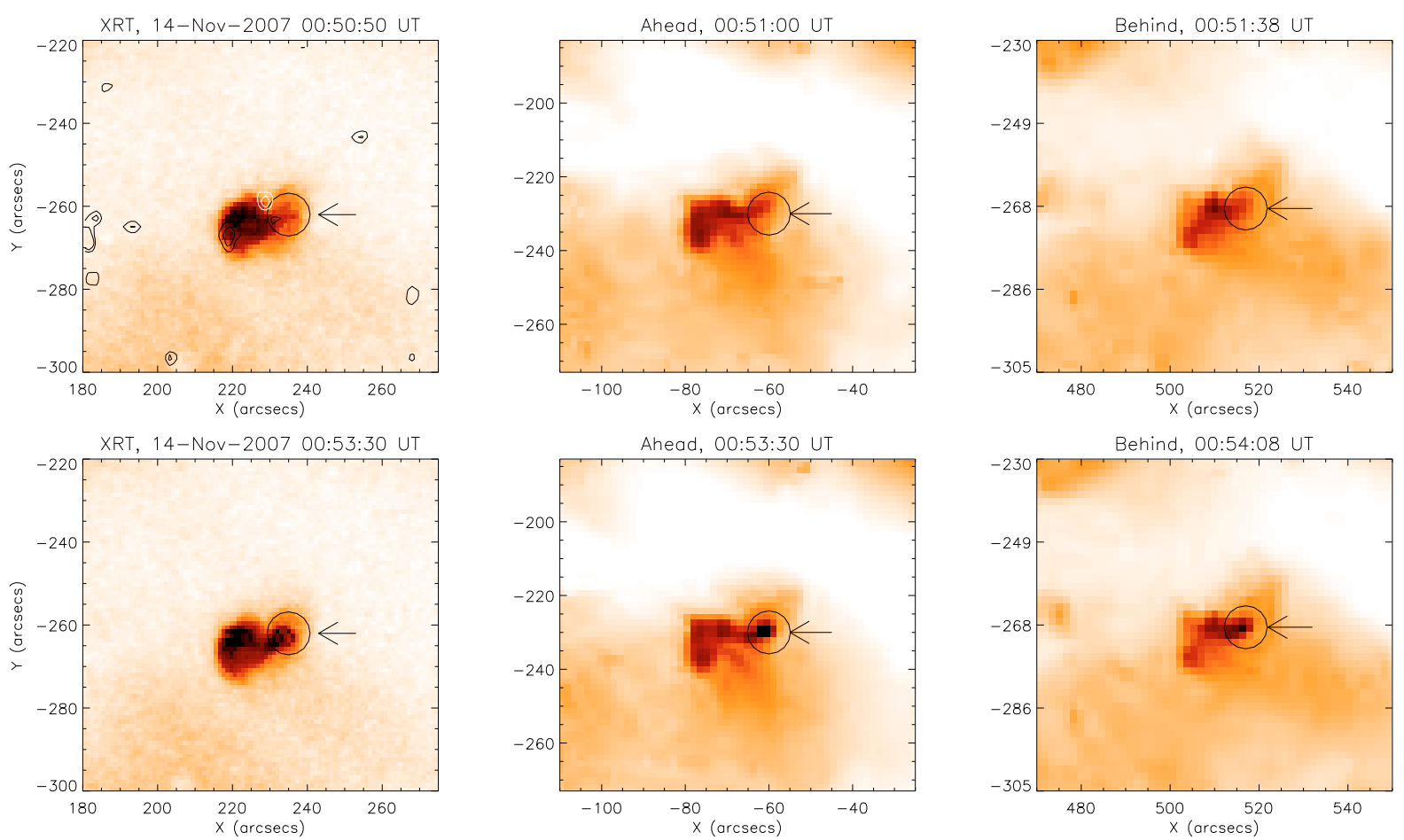

Fig. 2. From left to right: XRT and EUVI A and B images. The top row shows the images before the first micro-flare took place, while the bottom row presents the images taken seconds after it happened. The arrows point at the region where the micro-flare (surrounded by a circle) occurred. The XRT image (top left) is superimposed with the magnetic field contours at levels $-100,-50,50$ and $100 \mathrm{G}$ obtained from the magnetogram at 00:01:01 UT.

feature. The X-ray telescope was monitoring an equatorial coronal hole region together with the surrounding quiet Sun during several hours on 2007 November 14 (for more information on the data see Sect. 2). The EIS slit was scanning the area in the XRT field-of-view in west-east direction crossing the boundary of an equatorial coronal hole while the SUMER slit was in a sitand-stare mode and positioned in respect to the EIS field-of-view as shown in Fig. 1. The SUMER and EIS slits were pointing at roughly the same position at around 01:02 UT (no more than $1^{\prime \prime}-2^{\prime \prime}$ offset or none).

\subsection{Dynamics analysis and results}

We produced an animated sequence of XRT images (see the online movie) which indisputably revealed that the studied feature (Fig. 1) is a typical example of a so-called X-ray jet from a coronal BP (Shibata et al. 1992). The event started with a sudden brightening in an area of 3-4 $\operatorname{arcsec}^{2}$ size in a pre-existing coronal bright point at 00:52:50 UT and was registered by all three imagers - XRT, EUVI A and B (Fig. 2). We identified this region as the so-called micro-flare region where the energy deposition took place. After producing a sequence of difference images (Fig. 4) by subtracting from each image the precedent one, we were surprised to discover that an energy deposition, most probably from magnetic reconnection, happened several times during the event. We were able to identify five bursts during around 16 min. This can be seen in Fig. 4 on the images marked with stars. We note that although more darkenings (a sudden brightening corresponds to a darkening in a difference image) are seen, many of them come from plasma flows as determined by the spectroscopic observations (see below) which create bright and dark patches in the difference images. The energy deposition seems to occur in different places.
The BP appeared enlarged (in three dimensions corresponding to an expansion) on the XRT images, $40 \mathrm{~s}$ after the first energy deposition took place, while loops are clearly seen to expand 4 min later, i.e. around 00:56:50 UT, after the second and apparently larger energy deposition (Fig. 4). The first clearly distinguishable expanding loops can be seen in the XRT difference image around 00:57 UT in Fig. 4 and in the online movie. Following the event in EUVI A (B as well but at lower cadence) images we can clearly see that a large number of loops took off from the BP forming a plasma cloud, which moved away from the source region (see the EUVI A image sequence in the online movie, after 01:03 UT).

In Fig. 5 we show the spectra of the He II $256.34 \AA$ and Fe XII 195.12 A lines obtained from 00:56:33 UT, i.e. 4 min after the energy deposition took place. The top row in the figure displays the XRT images taken approximately in the middle of the EIS exposure duration. The corresponding EIS slit positions are overplotted. At 00:56:33 UT is the first registration of the jet-like event in EIS as blue-shifted emission. Note that SUMER was not yet observing at this time. The expanding plasma is only seen in the high-temperature lines as, for instance, Fe XII (up to Fe XV). No indication of cooler plasma is seen until 00:57:35 UT in Si VII $\left(\log T_{\max }=5.8 \mathrm{~K}\right)$ and until 00:58:37 UT in He II $\left(\log T_{\max }=4.7 \mathrm{~K}\right.$, note that the blue wing of He II is free from blends). After comparing this observation with the animation sequence, we can speculate on two possible scenarios: one is that the hotter and taller loops of the BP started first to expand and were followed by the lower lying cooler loops 2 min later. The other scenario is that the plasma from the energy deposition site is first propelled along the newly reconnected open magnetic field lines. The latter is consistent with the observation that a strong plasma outflow was registered along the straight magnetic field lines situated in the north-west 
M. S. Madjarska: Properties of X-ray jets
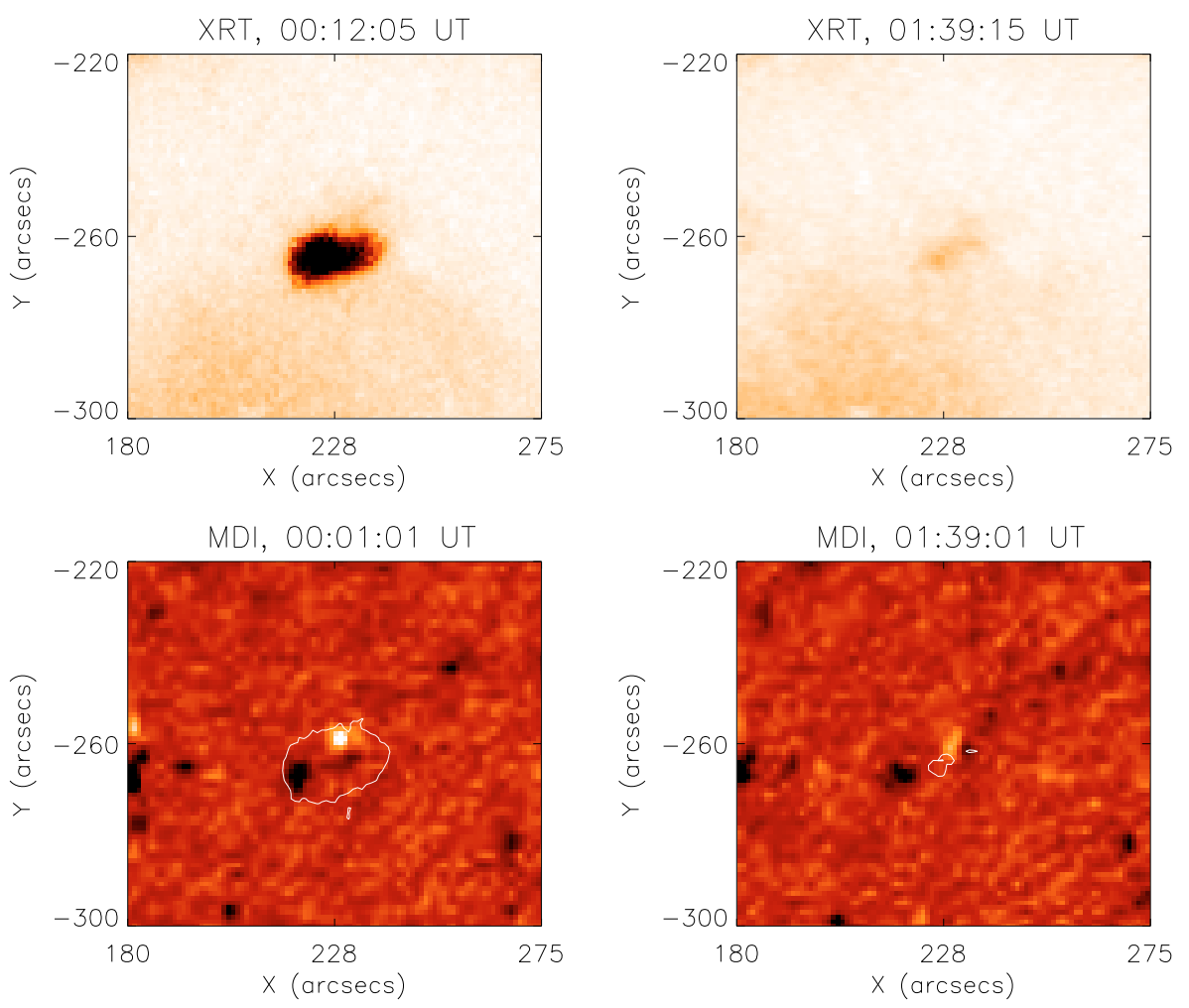

Fig. 3. Top: XRT images of the coronal bright point associated with the jet-like event. Bottom: MDI magnetograms scaled from -100 to $100 \mathrm{G}$ with the contour line of the coronal bright point seen in XRT overplotted as shown above. side of the jet-like event as seen in projection on the disk. There is also the possibility that these elongated structures represent loop legs that became quasi-open, i.e. they are not open into the interplanetary space. As can be seen in Fig. 5, in 4 min the plasma was accelerated from $130 \mathrm{~km} \mathrm{~s}^{-1}$ to $310 \mathrm{~km} \mathrm{~s}^{-1}$ derived from a double Gauss-fit of the Fe XII line. The Doppler shifts of the escaping loops are reaching up to $400-450 \mathrm{~km} \mathrm{~s}^{-1}$ (blueshifted emission). No falling-back plasma (red-shifted emission) was observed in the location of the jet.

We present the observations of XRT, EUVI/SECCHI, SUMER, and EIS obtained as close as possible in time in a composite figure (Fig. 6). The timing of the data corresponds to the moment when both spectrometers were staring at the same position on the solar disk at around 01:02 UT. The same combination of data for another six pointing times are available in the electronic edition of the journal (Figs. 11 to 16). Note that in spectrometers the time of a spectrum is defined as the time of the beginning of the exposure, while the time of an image is defined with the end of the exposure. The appearance of the event in all three imagers is quite different because of their different view angles. This multi-dimensional view of the studied phenomenon is of great importance for the understanding of the spectroscopic imprints of the studied event.

From the spectroscopic observations we established two main flows: a very fast outflow, which regenerates after each energy deposition and a down-flow guided along curved (most probably closed) magnetic field lines (best seen in EUVI B). Since SUMER started observing only at 01:01:19 UT, the dynamics in the transition region O V $629 \AA$ and N V $1238 \AA$ lines was caught after this time with strong blue-shifted emission, which expands beyond the spectral windows. Two sources seem to produce these Doppler shifts as judged from their appearance (Fig. 6): one confined in a small area of $2^{\prime \prime}-3^{\prime \prime}$ producing a strong emission that we associated with the north-west ray-like feature, and the other, more diffuse, cloud like emission, which could have been produced by the surrounding plasma pushed away by the shock generated from the energy deposition site or/and the expanding loops.

The energy deposition at 01:04 UT produced fast rising loops (Fig. 12) that generate Doppler shifts of up to $250 \mathrm{~km} \mathrm{~s}^{-1}$. It is clearly evident that the SUMER slit cuts through the legs of these rising loops. The emission from the coronal line yet again displays an additional line shift, which reflects the plasma stream along open field lines seen in the XRT image. The picture of the outflowing loops persists in the next two exposures at 01:04 UT and 01:05 UT, shown in Figs. 13 and 14. The hot outflow is not seen anymore in EIS as the EIS slit moves away from it (it has to be kept in mind that the EIS slit was scanning the area, while the SUMER slit was in a sit-and-stare position). After the last energy deposition at 01:05:30 UT, an outflow (the strongest in terms of emissivity) is observed only along the north-west part of the event.

The continuous down-flow shows Doppler shifts of up to 25-50 $\mathrm{km} \mathrm{s}^{-1}$ in the SUMER transition region lines and up to $150 \mathrm{~km} \mathrm{~s}^{-1}$ in the EIS coronal lines (Fig. 15). As the EIS slit is moving across the energy deposition site, the red-shift persists, although at 01:06 UT a blue-shifted component in the He II line is well distinguishable. From the available data we cannot speculate where this blue-shifted emission comes from.

The lifetime of the entire process of jet formation and evolution was approximately $27 \mathrm{~min}$ from the moment the first energy deposition was registered until a jet-like structure fully disappeared in the imagers. The dynamic phase of the jet from the moment it was first registered as an outflow until no signature of outflow is seen in the SUMER spectral lines is $18 \mathrm{~min}$. The down-flow persisted 2-3 min longer.

In Fig. 8 we present the line profiles of several spectral lines. The profiles were produced from the jet region with very high velocity. We selected only spectral lines that are free from nearby spectral lines on the blue side of the spectrum. The He II line is free of blends, but it is very possible that the blending hot lines make some contribution to the emission of the He II and 

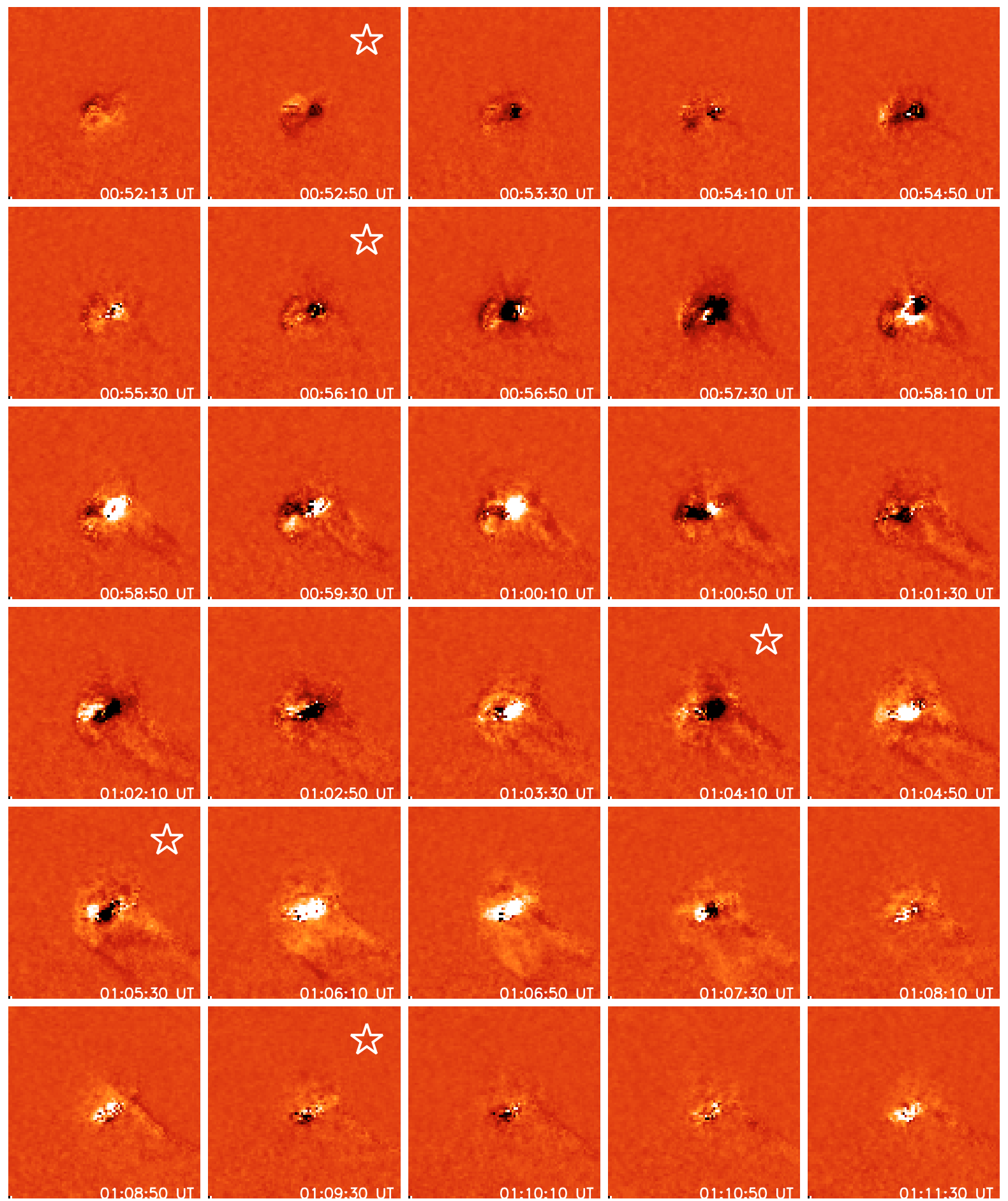

Fig. 4. Sequence of XRT difference images with overplotted stars, which indicate the moments of energy release.

its red wing. The $\mathrm{O}$ VI line at $184.12 \AA$ is also producing strong blue-shifted emission, but this emission is blended by another $\mathrm{O}$ VI line at $183.94 \AA$. The later line is usually up to twice as weak as O VI $184.12 \AA$ and therefore the emission in excess would come from the blue wing of O VI $184.12 \AA$. The comparison of the spectral lines Fe VIII, X, XII and XIII shows an increase of the emission with the increase of the formation temperature of the spectral line, suggesting that the amount of hotter plasma dominates the outflow. The Doppler shift of the emitted plasma estimated from a double Gauss fit is similar, e.g. $246 \mathrm{~km} \mathrm{~s}^{-1}$, $259 \mathrm{~km} \mathrm{~s}^{-1}, 279 \mathrm{~km} \mathrm{~s}^{-1}$ and $265 \mathrm{~km} \mathrm{~s}^{-1}$ for Fe VIII, X, XII and XIII, respectively. Unfortunately, the low signal-to-noise ratio of the higher ionisation lines does not permit us to investigate their line profiles in this region.

The magnetograms show a bipolar magnetic region of maximum -153 and $172 \mathrm{G}$ at 00:01:01 UT and -160 and $63 \mathrm{G}$ at 01:39:01 UT concentrated in a single pixel surrounded by a weaker magnetic field. The negative polarity covers a larger area and is separated from the positive polarity by $12^{\prime \prime}$ at both times. A weaker negative polarity of around $-50--60 \mathrm{G}$ is seen next to the positive polarities in both times. The low spatial resolution and poor cadence of the available MDI magnetograms do 
M. S. Madjarska: Properties of X-ray jets
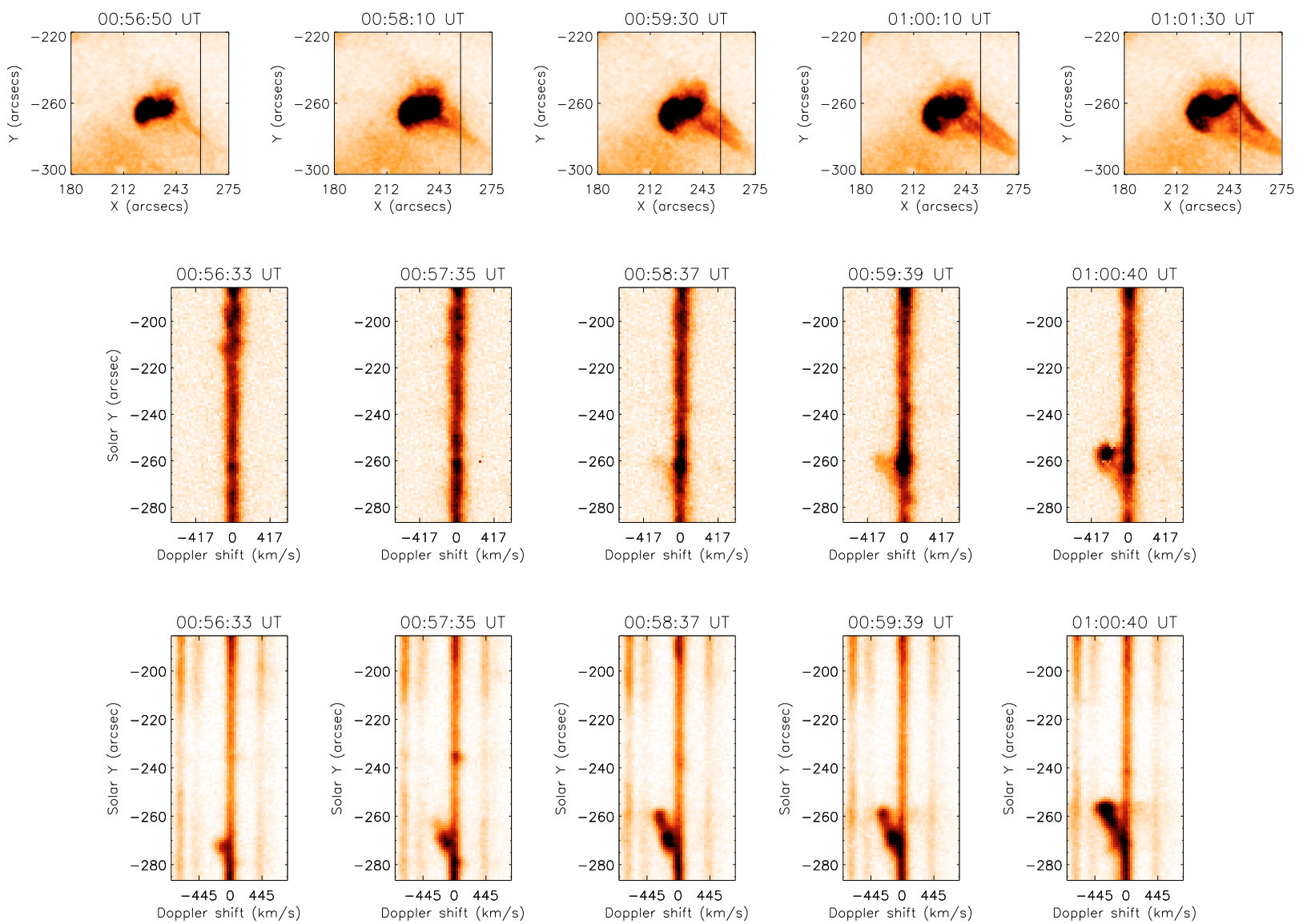

Fig. 5. XRT images (top) of the jet-like event shortly after it started. EIS He II $256.32 \AA$ (middle) and Fe XII $195.12 \AA$ (bottom) spectra along the EIS slit registered around the time the XRT images were taken. Note that the timing of these images is before EIS reaches the SUMER slit position. The slit moves in west-east direction with a step of $2^{\prime \prime}$ at each exposure.
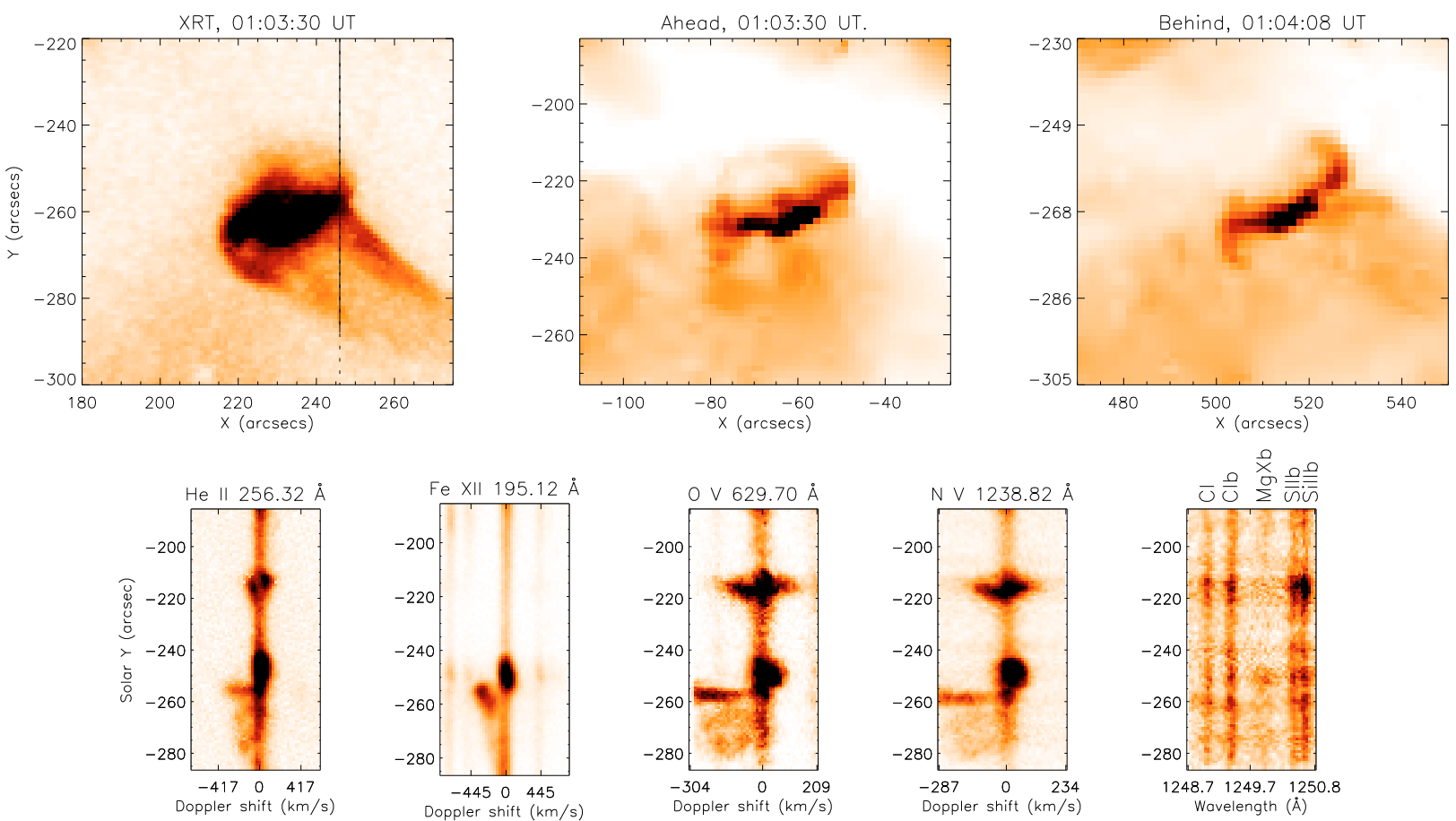

Fig. 6. Top: XRT image with overplotted SUMER slit position and EUVI A and B images during the jet-like phenomenon. Bottom (from left to right): EIS He II $256.32 \AA$ and Fe XII $195.12 \AA$ spectra along the EIS slit at 01:02:44 UT; SUMER O V and N V spectra along the SUMER slit; and a spectral window comprising C I 1248.0 A, C I $1249.0 \AA$, Mg x $624.9 \AA$ taken in second order, Si II $1250.41 \AA$ and S II $1250.58 \AA$ (for details on blends and formation temperatures consult Table 1) at 01:02:019 UT. 

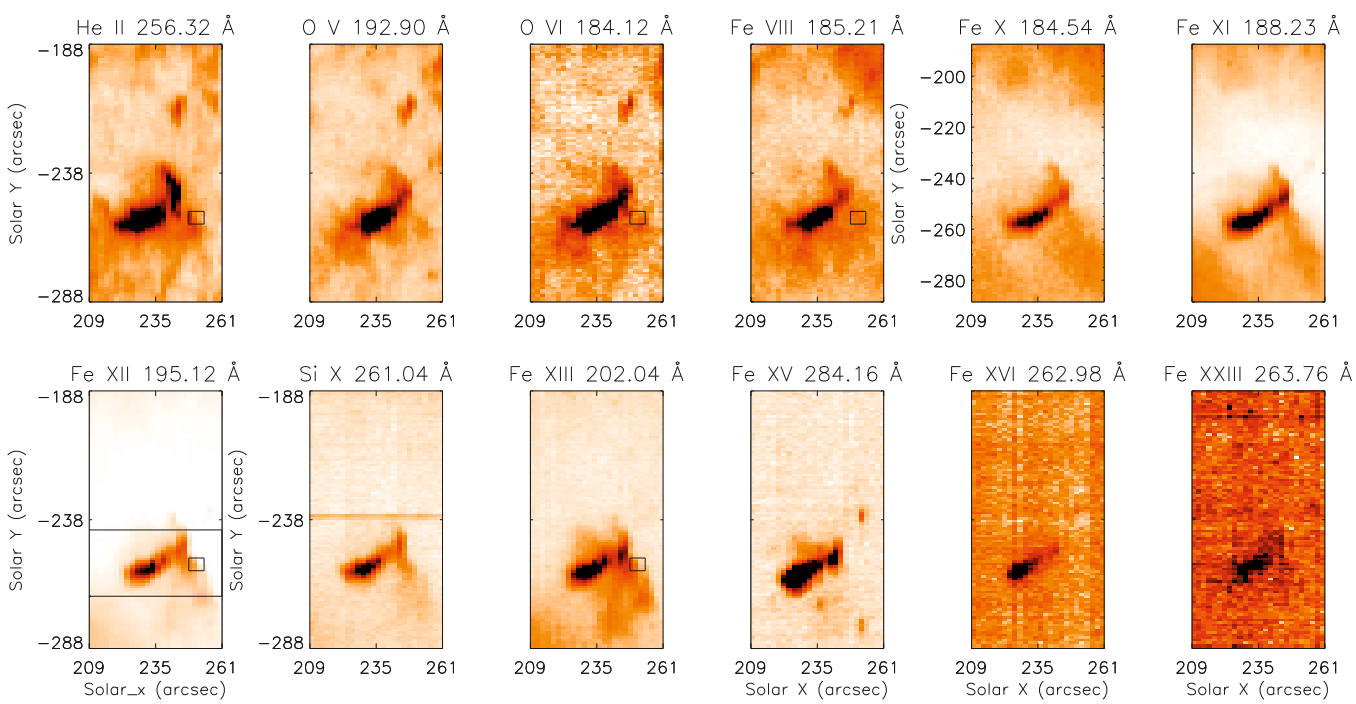

Fig. 7. EIS rasters taken in 12 spectral lines with formation temperatures ranging from $50000 \mathrm{~K}$ to $12 \mathrm{MK}$ assuming ionisation equilibrium. The overplotted box of the Fe XII raster denotes the field-of-view shown in Fig. 9. Details on the formation temperature of each line can be found in Table 2.
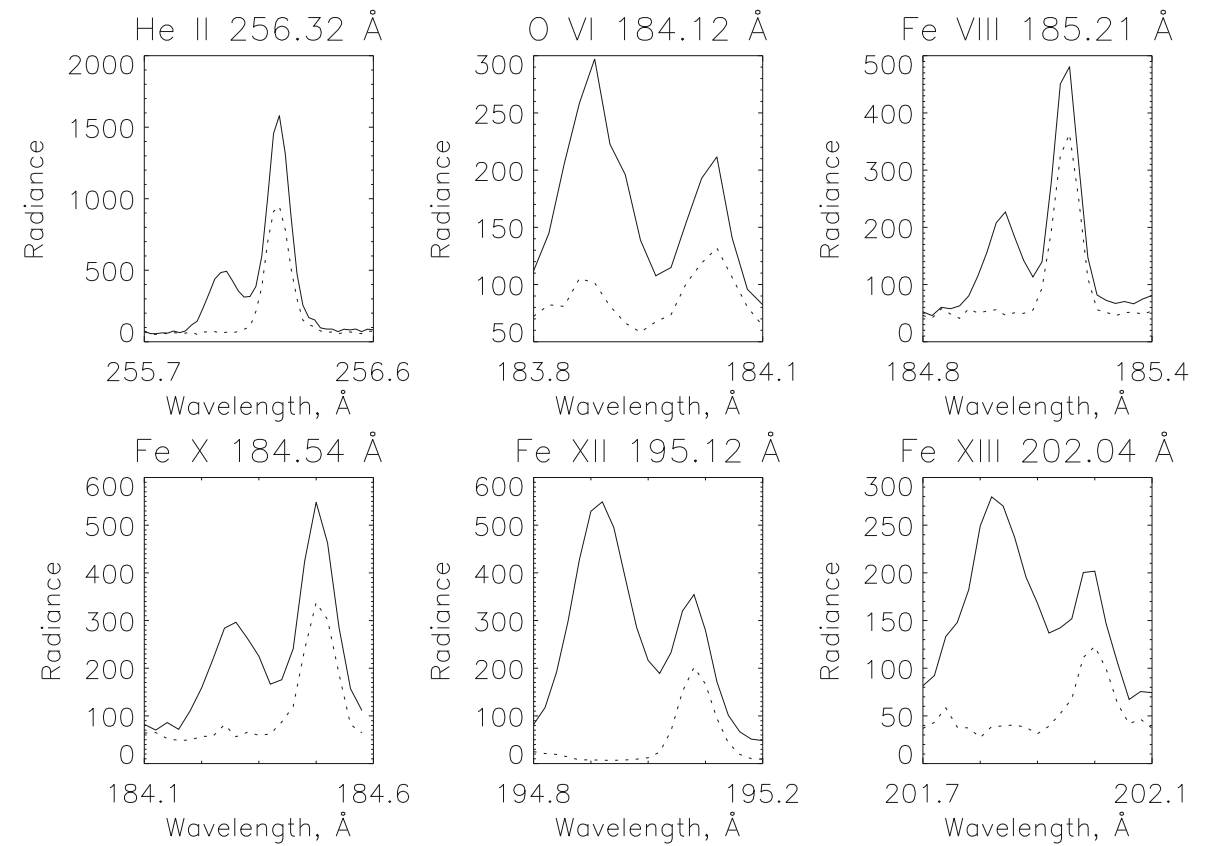

Fig. 8. EIS line profiles taken from the region shown with a small box in Fig. 7. The radiance is in units erg $\mathrm{cm}^{-1} \mathrm{~s}^{-1} \mathrm{sr}^{-1} \AA^{-1}$. The dotted line corresponds to a reference profile.

not permit any further study that could add valuable information to our analysis. The decrease in the positive polarity suggests that the magnetic energy associated with it was converted into heat and kinetic energy responsible for the observed hightemperature plasma and high-velocity plasma outflow, respectively. In Fig. 3 we show the two magnetograms in which the XRT image contours taken at the closest times are overplotted.

\subsection{Temperature analysis and results}

That brings us to the temperature analysis of the emitting plasmas. We can separate the jet into three main temperature regions: the energy deposition site, the up-flowing (blue-shifted emission) plasma of the jet-like event and the down-flow (red-shifted emission). The micro-flaring site emits in a large temperature range from $0.5 \mathrm{MK}$ (He II) to up to $12 \mathrm{MK}$ (Fe XXIII $263.76 \AA$ ) (Fig. 7). For the first time a plasma temperature of $12 \mathrm{MK}$ was detected in a quiet Sun region. The Fe XXIII $263.76 \AA$ line was first identified in observations with the Naval Research Laboratory spectrograph during a two-ribbon flare by Widing (1975). It was also reported by Del Zanna (2008) in a flare observed with EIS. On the blue side of the line is Ar XV $263.69 \AA$, which can easily be separated from Fe XXIII with a double Gauss-fit.

The outflowing plasma has a temperature from 0.5 MK (He II) to $2 \mathrm{MK}$ (Fe XV) as already reported by Culhane et al. (2007b). The authors used slot observations that carry some uncertainties because of line blends in the observed spectral windows. However, there is a possibility of even higher temperature plasma, which may not be detectable in EIS observations because of the weakness of the higher ionisation lines like for instance Fe XVI and Fe XVII. No signature was detected in the SUMER chromospheric lines that are formed at temperatures as low as $10000 \mathrm{~K}$ (C I; see Table 1 for more details). 

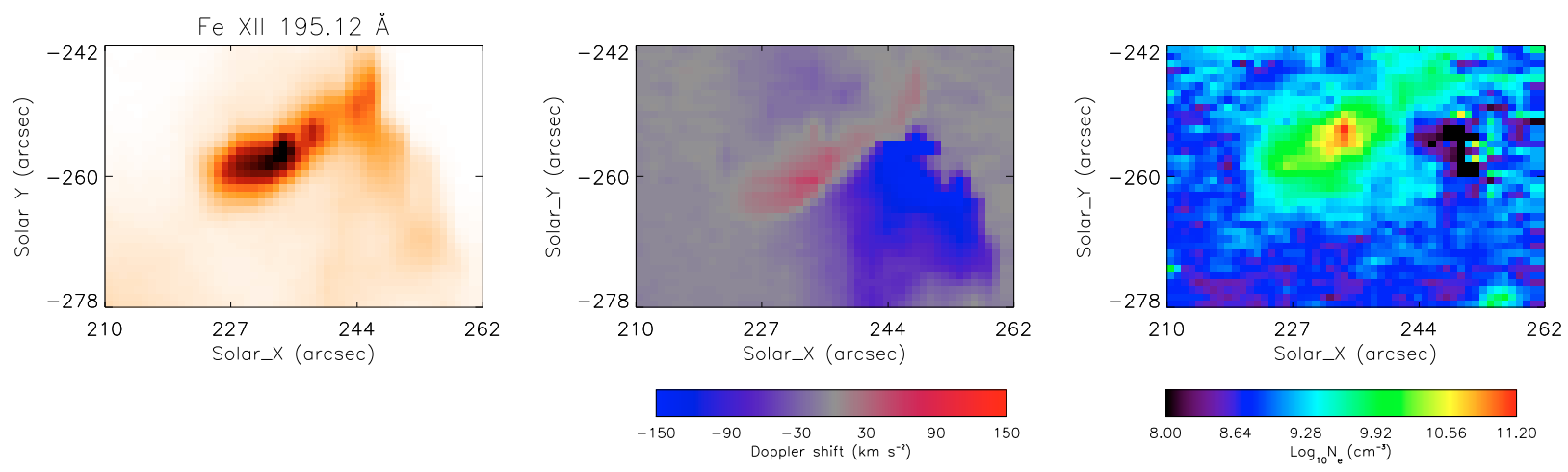

Fig. 9. Top: partial view of the radiance image in the Fe XII line shown in Fig. 7. Bottom: an electron density image derived from the Fe XII line ratio (see Sect. 3.3 for more details).

The third region that corresponds to the down-flowing plasma seen as red-shifted emission has temperatures ranging from $15000 \mathrm{~K}$ (S II) to $1.3 \mathrm{MK}$ (Fe XII and Si X). That clearly suggests a cooler plasma downstream.

We need to point out a problem concerning an uncertainty in using the SUMER Mg X $624.9 \AA$ line for coronal diagnostics. If we compare the simultaneous EIS and SUMER observations shown in Fig. 6, the very weak response of $\mathrm{Mg} \mathrm{X}$ is clearly evident, while Fe XII, which is formed at a similar temperature, has a very strong increase. The $\mathrm{Mg} \mathrm{X}$ response in the outflow is completely absent and shows only a very modest increase in the energy deposition region. In the past this spectral line was used to determine coronal response of transient features in the quiet Sun with a main conclusion that most of these phenomena do not reach coronal temperatures because of the lack of emission in the $\mathrm{Mg} \mathrm{X}$ line. This, consequently, questioned their direct contribution to coronal heating. The count rate in the $\mathrm{Mg}$ X $624.9 \AA$ line as observed in second order of diffraction is 0.17 counts/s, and if we assume a minimum $10 \%$ contribution from the blending Si II $1250.09 \AA$ and another few percent from other lines (see for more details Teriaca et al. 2002), we arrive at 0.15 counts/s. In comparison, the count rate for the Fe XII line is 6.17 counts/s, which gives a ratio of the two lines (Fe XII/ Mg X) of around 40. How can this discrepancy be explained? Golub et al. (1989) found that a possible reason for the lower variability of coronal emission in $\mathrm{Mg} \mathrm{X}$ can be caused by very slow ionisation and recombination time scales. Although, it may take $\mathrm{Mg} \mathrm{X}$ several hundred seconds to reach ionisation equilibrium (assuming $N_{\mathrm{e}}=10^{9} \mathrm{~cm}^{-3}$ ), $\mathrm{Mg}$ X should still be very strong at the $1.5-2 \mathrm{MK}$ even in transient ionisation conditions (J. G. Doyle, priv. comm.). We investigated the $\mathrm{Mg}$ X emissivity using the atomic database CHIANTI v6.0 with ionisation fractions from Mazzotta et al. (1998) and found that for a quiet Sun DEM (differential emission measure) and active region DEM (if we assume a bright point to be a mini active region), the $\mathrm{Mg} \mathrm{X}$ line is approximately six times weaker than Fe XII. That still does not explain the lack of counts in the $\mathrm{Mg} \mathrm{X}$ line. The other possible reason is a low SUMER sensitivity for observations in second order of diffraction.

\subsection{Density analysis and results}

We also calculated the electron density of the jet-like event and its footpoints, which comprise the bright point and the microflaring site therein. From the analysis of density sensitive line pairs available in our dataset, we concluded that the only line ratio that could provide reliable density diagnostics is the line ratio of Fe XII 186.88 to $195.12 \AA$. The Fe XIII ratio of 203.82 to 202.04 is not suitable because of discrepancies in estimating densities above $10^{10} \mathrm{~cm}^{-3}$ (for more details see Young et al. 2009). Equally, the blend in the blue wing of the Fe XIII $203.82 \AA$ line makes the use of this line ratio quite uncertain for events showing blue-shifts as in the present case.

There are several blending issues concerning the Fe XII 186.88 and $195.12 \AA$ lines. A comprehensive study on this can be found in Young et al. (2009). The theoretical line ratio derived with CHIANTI v6.0 includes for $186 \AA$ the two Fe XII lines at $186.85 \AA$ and $186.88 \AA$ and for $195 \AA$ the two Fe XII lines $-195.12 \AA$ and $195.18 \AA$. The Fe XII 186 is also blended by S XI $186.84 \AA$, which was taken as $19.5 \%$ of S XI $191.27 \AA$ (Young et al. 2009). Note that in the high-velocity part of the jet the density values are unreliable because of the blend in the far blue wing of Fe XII $186.88 \AA$ by Fe VIII $186.60 \AA$. In Fig. 9 we show a combination of the radiance, Doppler shift (from a single Gauss fit) and electron density images of the event. We obtained an average density value for the BP region of approximately $4 \times$ $10^{9} \mathrm{~cm}^{-3}$, a value of around $4 \times 10^{8} \mathrm{~cm}^{-3}$ for the surrounding corona and a maximum value of $4 \times 10^{10} \mathrm{~cm}^{-3}$ for the density of the micro-flaring region at temperature $1.3 \times 10^{6} \mathrm{~K}$, which is the maximum of the formation temperature of Fe XII.

\section{Discussion and conclusions}

\subsection{The physical parameters}

The physical parameters of X-ray jets were the subject of several studies (Shimojo \& Shibata 2000; Culhane et al. 2007b; Savcheva et al. 2007; Doschek et al. 2010). Shimojo \& Shibata (2000) derived the temperature and the density of X-ray jets from Yohkoh/SXT observations by analysing 16 jets and their footpoints. The jet temperatures were estimated to be in the order of 3-8 MK and the density in the range from 0.7 to $4.0 \times 10^{9} \mathrm{~cm}^{-3}$. The authors found that the temperatures of the footpoint microflares were similar to those of the jet, i.e. 4-8 MK, while the densities were slightly higher, e.g. $2.4-10.0 \times 10^{9} \mathrm{~cm}^{-3}$. The first to deliver results on jet plasma quantities from Hinode observations were Culhane et al. (2007b). They used slot observations which can also give only a rough estimate of the physical quantities. For the two analysed jets, the temperatures ranged from 0.4 MK to 5.0 MK, while the density was $4 \times 10^{9} \mathrm{~cm}^{-3}$. The most recently published values by Doschek et al. (2010) are $0.3-2.2 \mathrm{MK}$ for the temperature and approximately $10^{9} \mathrm{~cm}^{-3}$ or less for the electron density. In comparison, we found in the 
footpoints of the jet, i.e. at the location of the micro-flare, a temperature (derived as the maximum of the formation temperature of the observed spectral line) of up to $12 \mathrm{MK}$, while the density was $4 \times 10^{10} \mathrm{~cm}^{-3}$. The average densities of the bright point where the outflow originates from, is $4 \times 10^{9} \mathrm{~cm}^{-3}$ (at a temperature of $1.3 \times 10^{6} \mathrm{~K}$ ), which is comparable to earlier studies.

\subsection{Comparison with a $3 D \mathrm{MHD}$ model}

We compared the observed dynamics and the physical parameters of the jet-like event analysed in the present paper with the most recent and very close-to-realistic simulated jetlike event by Moreno-Insertis et al. (2008). They developed a 3D MHD model based on the interaction of a twisted magnetic flux rope rising from the convection zone and expanding into the corona. A current sheet forms between the counter-aligned side of the flux tube with the ambient coronal field. The authors describe the formation of a "double-chambered" vault-shaped region formed of current loops that are both emerged and reconnected. The on-disk view of our jet does not permit us to make any comparison with these structures, but similar structures were already described in the literature. In the simulated jet two regions of reconnected field lines are formed: first are the reconnected open field lines, which is comparable to what we see shortly after the first energy deposition took place, followed by closed loops rising from below. In comparison with the simulated feature, we clearly distinguish the formation of a high-velocity outflow along open magnetic field lines followed very shortly by the expulsion of numerous loops which, however, is not seen in the simulations. Recently it has been found by Subramanian et al. (2010) that X-ray jets are always associated with pre-existing or newly emerging (at X-ray temperatures) coronal bright points. They concluded that a necessary condition for the generation of outflows is the interaction of closed and open magnetic field lines. Therefore, there are always pre-existing loop structures, i.e. coronal bright points, which are expelled during the event. There is also the possibility that some of the expelled loops represent newly reconnected loops, but we strongly believe that most of the observed loop structures lifting away belong to the pre-existing bright point. One strong argument for this is that the BP almost fully disappears at X-ray temperatures after the jet-like event. A careful check of the lower temperature images (TRACE and EUVI Fe IX/X $171 \AA$ ) that include cooler spectral lines (transition region temperatures) indicated that there was still a remaining lower lying BP, which consequently produced another jet, but only at low temperatures, until the BP fully disappeared. This scenario was observed in the majority of the BP-producing jets in Subramanian et al. (2010), but it will be a subject of a forthcoming article.

Can other mechanism(s) instead of the flux emergence trigger magnetic reconnection in BPs? That jet-like events occur predominantly at coronal hole boundaries and inside coronal holes where closed loop structures exist, and that coronal holes are rotating quasi-rigidly in respect to the surrounding quiet Sun (Timothy et al. 1975), suggests that the necessary conditions exist these two topologically different magnetic fluxes push together to form a current sheet and reconnect. We also detected for the first time several energy depositions during the phenomenon, which indicates that several episodes of reconnection may have taken place. This scenario needs to be explored theoretically.
The physical parameters obtained from the Moreno-Instertis et al. experiment compare very well with the results of the present study. The temperature of the reconnection site of $T \approx$ $3 \times 10^{7} \mathrm{~K}$ is very close to the $1.3 \times 10^{7} \mathrm{~K}$ derived here. The high plasma temperature was, however, not detected in the outflowing plasma, but that could be simply because of a low signal-to-noise ratio in the observed line, as discussed earlier. Both the velocity of the plasma and the duration of the event are quite alike.

As we mentioned earlier, the event described here was selected from a large number of events registered during a coordinated campaign of Hinode and SoHO. A forthcoming paper will give details on the dynamics and physical parameters of large number of events identified by Subramanian et al. (2010).

Acknowledgements. I would like to acknowledge the important comments and suggestions of the anonymous referee. The author thanks ISSI, Bern for the support of the team "Small-scale transient phenomena and their contribution to coronal heating". The author thanks K. Galsgaard and J. G. Doyle for the careful reading and useful comments of this manuscript. Research at Armagh Observatory is grant-aided by the N. Ireland Department of Culture, Arts and Leasure. We also thank STFC for support via grants ST/F001843/1 and PP/E002242/1. Hinode is a Japanese mission developed and launched by ISAS/JAXA, with NAOJ as domestic partner and NASA and STFC (UK) as international partners. It is operated by these agencies in co-operation with ESA and NSC (Norway).

\section{References}

Archontis, V., Hood, A. W., \& Brady, C. 2007, A\&A, 466, 367

Brown, C. M., Feldman, U., Seely, J. F., Korendyke, C. M., \& Hara, H. 2008, ApJS, 176, 511

Cirtain, J. W., Golub, L., Lundquist, L., et al. 2007, Science, 318, 1580

Culhane, J. L., Harra, L. K., James, A. M., et al. 2007a, Sol. Phys., 243, 19

Culhane, L., Harra, L. K., Baker, D., et al. 2007b, PASJ, 59, 751

Del Zanna, G. 2008, A\&A, 481, L69

Doschek, G. A., Landi, E., Warren, H. P., \& Harra, L. K. 2010, ApJ, 710, 1806

Golub, L., Hartquist, T. W., \& Quillen, A. C. 1989, Sol. Phys., 122, 245

Golub, L., Deluca, E., Austin, G., et al. 2007, Sol. Phys., 243, 63

Handy, B. N., Acton, L. W., Kankelborg, C. C., et al. 1999, Sol. Phys., 187, 229

Heyvaerts, J., Priest, E. R., \& Rust, D. M. 1977, ApJ, 216, 123

Isobe, H., Tripathi, D., \& Archontis, V. 2007, ApJ, 657, L53

Jiang, Y. C., Chen, H. D., Li, K. J., Shen, Y. D., \& Yang, L. H. 2007, A\&A, 469, 331

Kamio, S., Curdt, W., Teriaca, L., Inhester, B., \& Solanki, S. K. 2010, A\&A, 510, L1

Karpen, J. T., Antiochos, S. K., Devore, C. R., \& Golub, L. 1998, ApJ, 495, 491 Lemaire, P., Wilhelm, K., Curdt, W., et al. 1997, Sol. Phys., 170, 105

Mazzotta, P., Mazzitelli, G., Colafrancesco, S., \& Vittorio, N. 1998, A\&AS, 133, 403

Moreno-Insertis, F., Galsgaard, K., \& Ugarte-Urra, I. 2008, ApJ, 673, L211

Nishizuka, N., Shimizu, M., Nakamura, T., et al. 2008, ApJ, 683, L83

Nisticò, G., Bothmer, V., Patsourakos, S., \& Zimbardo, G. 2009, Sol. Phys., 259, 87

Pariat, E., Antiochos, S. K., \& DeVore, C. R. 2009, ApJ, 691, 61

Patsourakos, S., Pariat, E., Vourlidas, A., Antiochos, S. K., \& Wuelser, J. P. 2008, ApJ, 680, L73

Savcheva, A., Cirtain, J., Deluca, E. E., et al. 2007, PASJ, 59, 771

Scherrer, P. H., Bogart, R. S., Bush, R. I., et al. 1995, Sol. Phys., 162, 129

Shibata, K., Ishido, Y., Acton, L. W., et al. 1992, PASJ, 44, L173

Shimojo, M., \& Shibata, K. 2000, ApJ, 542, 1100

Shimojo, M., Hashimoto, S., Shibata, K., et al. 1996, PASJ, 48, 123

Shimojo, M., Narukage, N., Kano, R., et al. 2007, PASJ, 59, 745

Subramanian, S., Madjarska, M. S., \& Doyle, J. G. 2010, A\&A, 516, A50

Teriaca, L., Madjarska, M. S., \& Doyle, J. G. 2002, A\&A, 392, 309

Timothy, A. F., Krieger, A. S., \& Vaiana, G. S. 1975, Sol. Phys., 42, 135

Tsuneta, S., Acton, L., Bruner, M., et al. 1991, Sol. Phys., 136, 37

Widing, K. G. 1975, ApJ, 197, L33

Wilhelm, K., Curdt, W., Marsch, E., et al. 1995, Sol. Phys., 162, 189

Yokoyama, T., \& Shibata, K. 1995, Nature, 375, 42

Yokoyama, T., \& Shibata, K. 1996, PASJ, 48, 353

Young, P. R., Del Zanna, G., Mason, H. E., et al. 2007, PASJ, 59, 857

Young, P. R., Watanabe, T., Hara, H., \& Mariska, J. T. 2009, A\&A, 495, 587

Pages 11 to 14 are available in the electronic edition of the journal at http://www . aanda.org 
M. S. Madjarska: Properties of X-ray jets
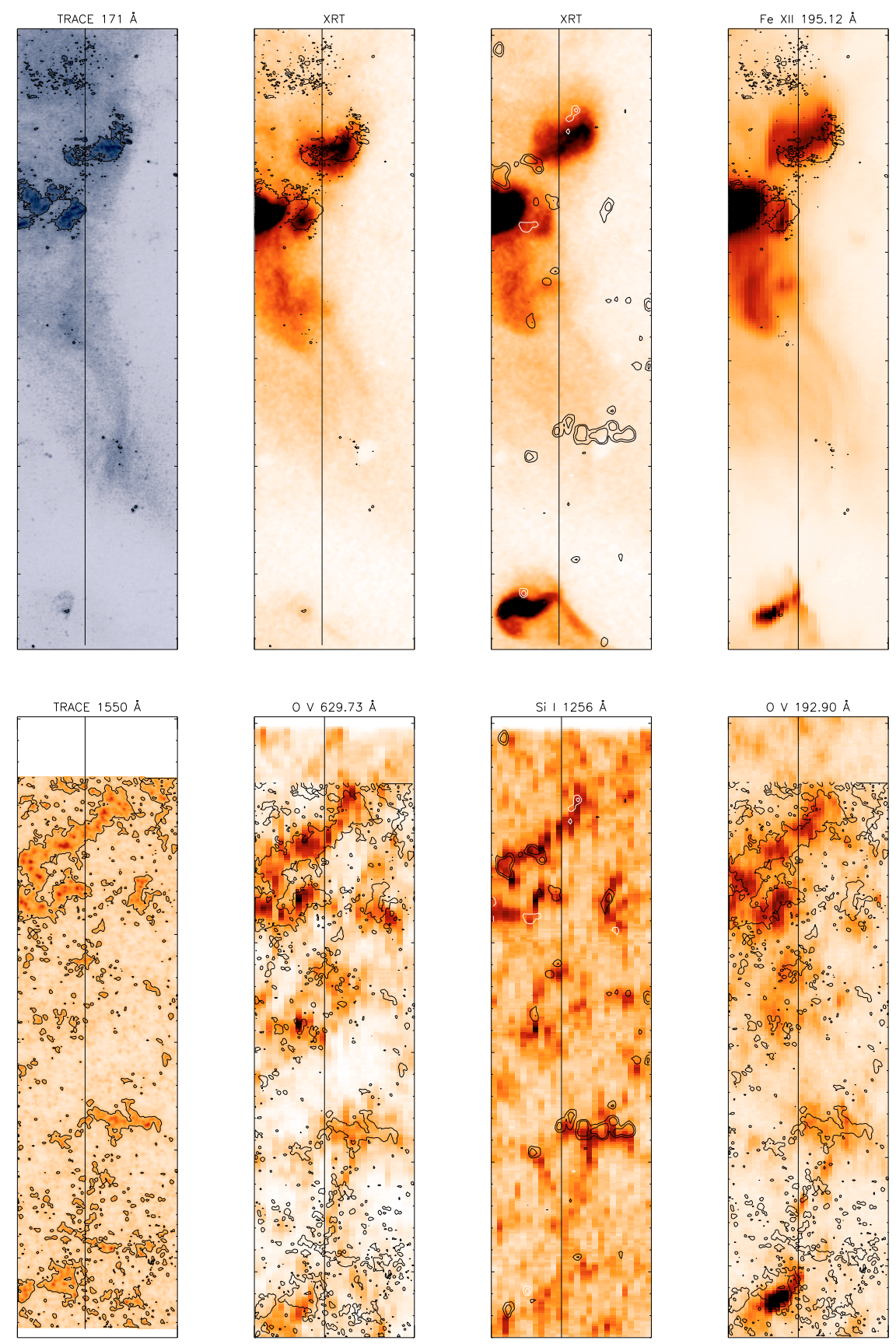

Fig. 10. Top, from left to right: TRACE $171 \AA$ image taken at 03:27 UT, XRT image at 03:27 UT, XRT image at 01:01 UT, EIS raster image in Fe XII 195.12 A obtained between 00:40 UT and 01:21 UT. The TRACE 171 $\AA$, the XRT image at 03:27 UT and the EIS image have the TRACE $171 \AA$ contour overplotted. The XRT image is overplotted with the $-100,-50,50$ and $100 \mathrm{G}$ contours of the magnetogram taken at 00:01:01 UT. Bottom, from left to right: TRACE $1550 \AA$ image taken at 03:28 UT, SUMER O V $629.73 \AA$ and S I $1256.49 \AA$ raster images taken between 02:43 UT and 02:56 UT, EIS O v 192.90 A raster taken from 00:40 to 01:21 UT. The SUMER Si I image is overplotted with the $-100,-50,50$ and $100 \mathrm{G}$ contours of the magnetogram taken at 00:01:01 UT. The rest of the images have the TRACE $1550 \AA$ contour superimposed. All the images (top and bottom) are overplotted with the SUMER slit position. The positive polarity ais shown in white and the negative in black colours. 

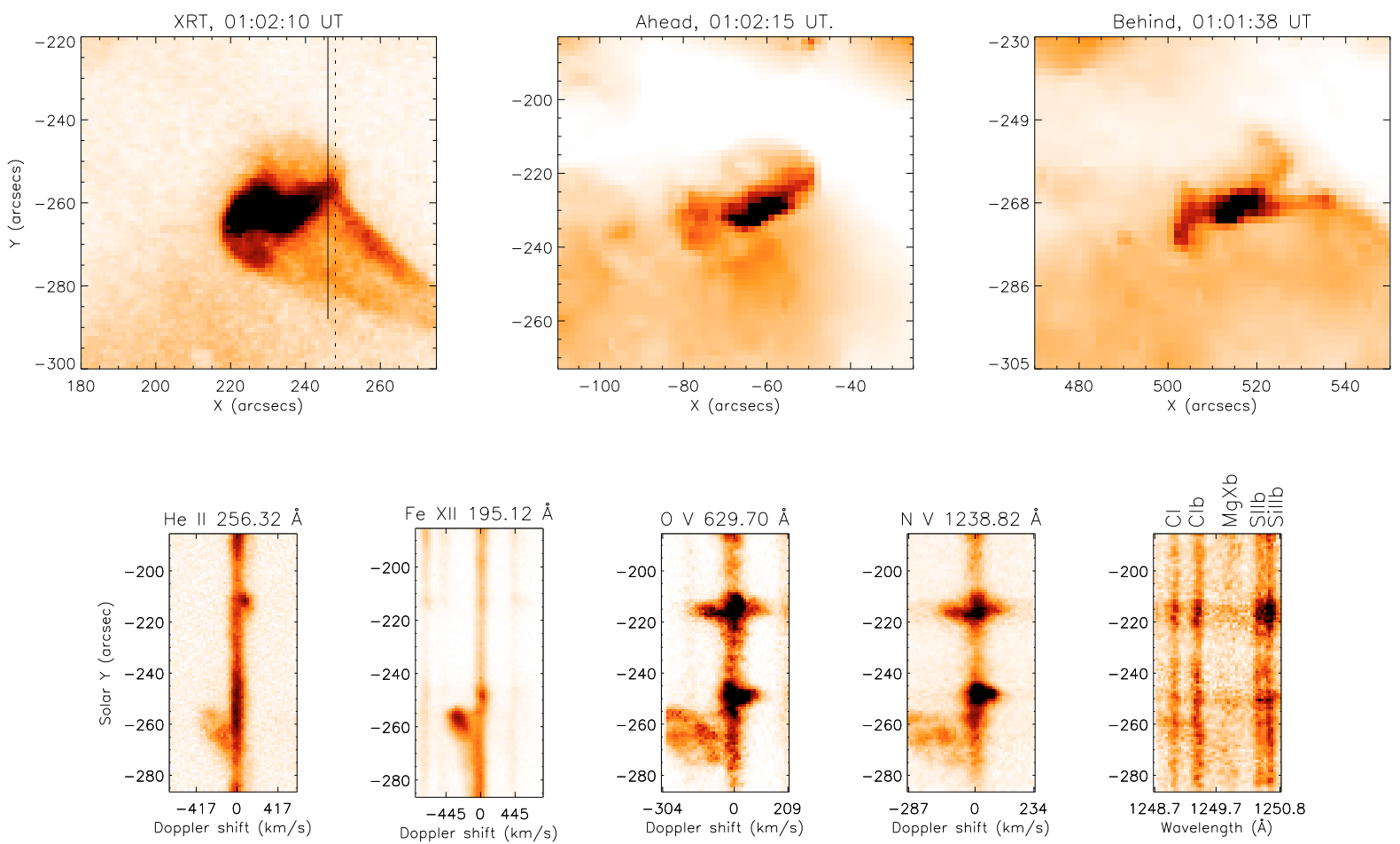

Fig. 11. Top: XRT image with superposition of the SUMER slit position, EUVI A and B images during the jet-like phenomenon. Bottom (from left to right): EIS He II 256.32 ̊ and Fe XII 195.12 A spectra along the EIS slit at 01:01:42 UT; SUMER O V and N V spectra along the SUMER slit; and a spectral window comprising C I 1248.0 A, C I 1249.0 ̊, Mg X $624.9 \AA$ taken in second order, Si II $1250.41 \AA$ and S II $1250.58 \AA$ (for details on blends and formation temperatures consult Table 1) at 01:01:19 UT. The temporal evolution of as seen in the to three panels is shown in the on-line movie.
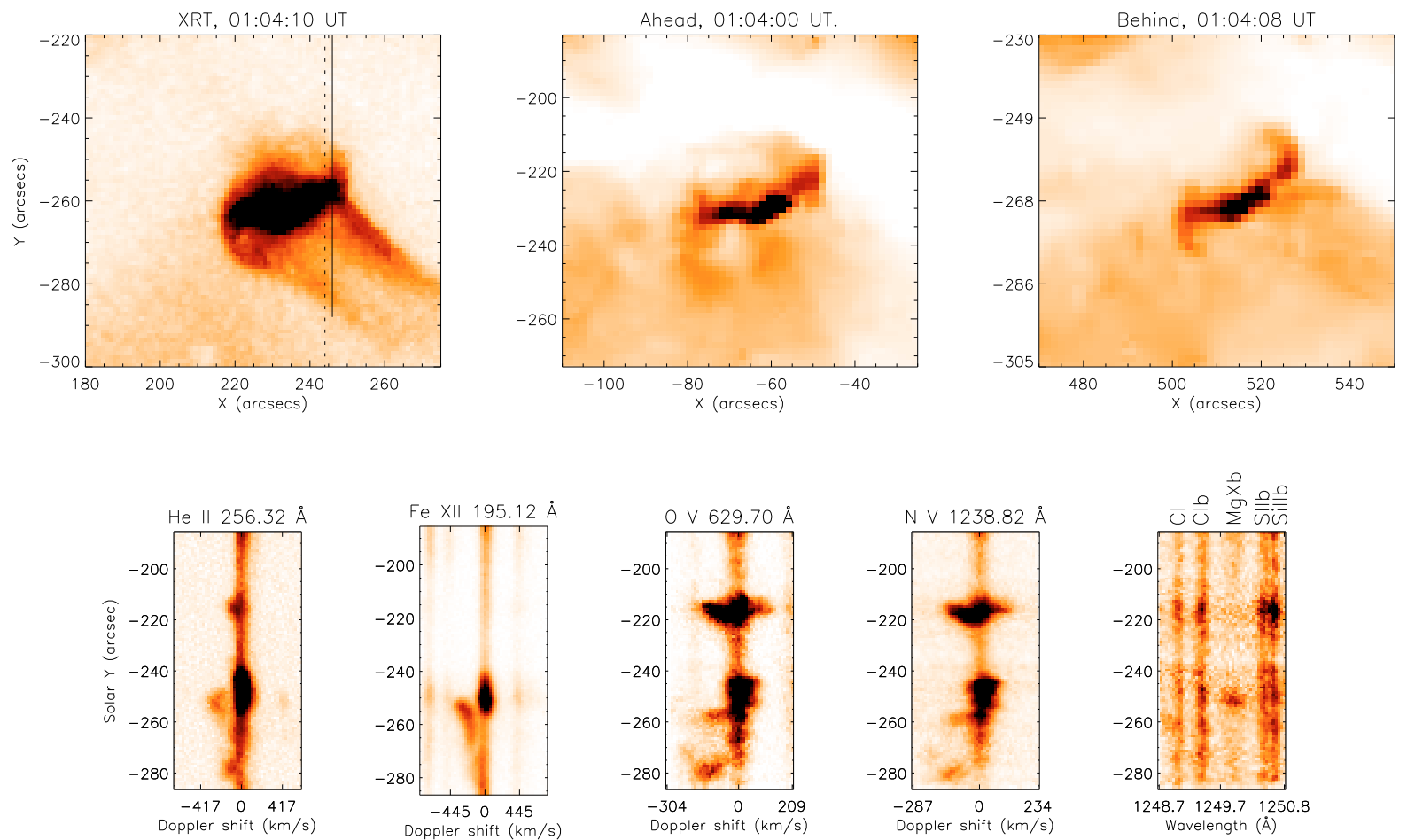

Fig. 12. The same as Fig. 11 with EIS at 01:03:46 UT and SUMER at 01:03:26 UT. 
M. S. Madjarska: Properties of X-ray jets
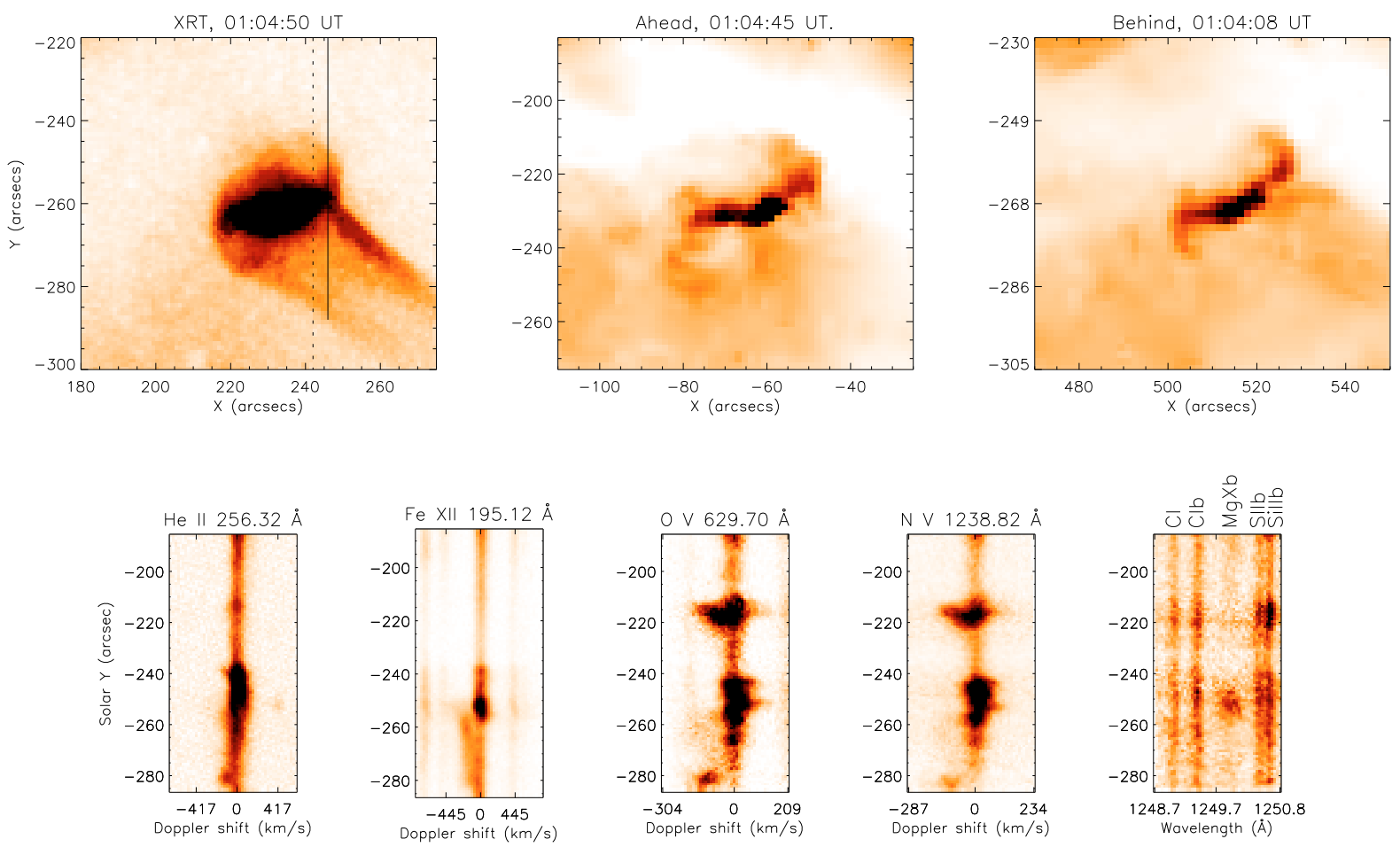

Fig. 13. The same as Fig. 11 with EIS at 01:04:48 UT and SUMER at 01:04:26 UT.
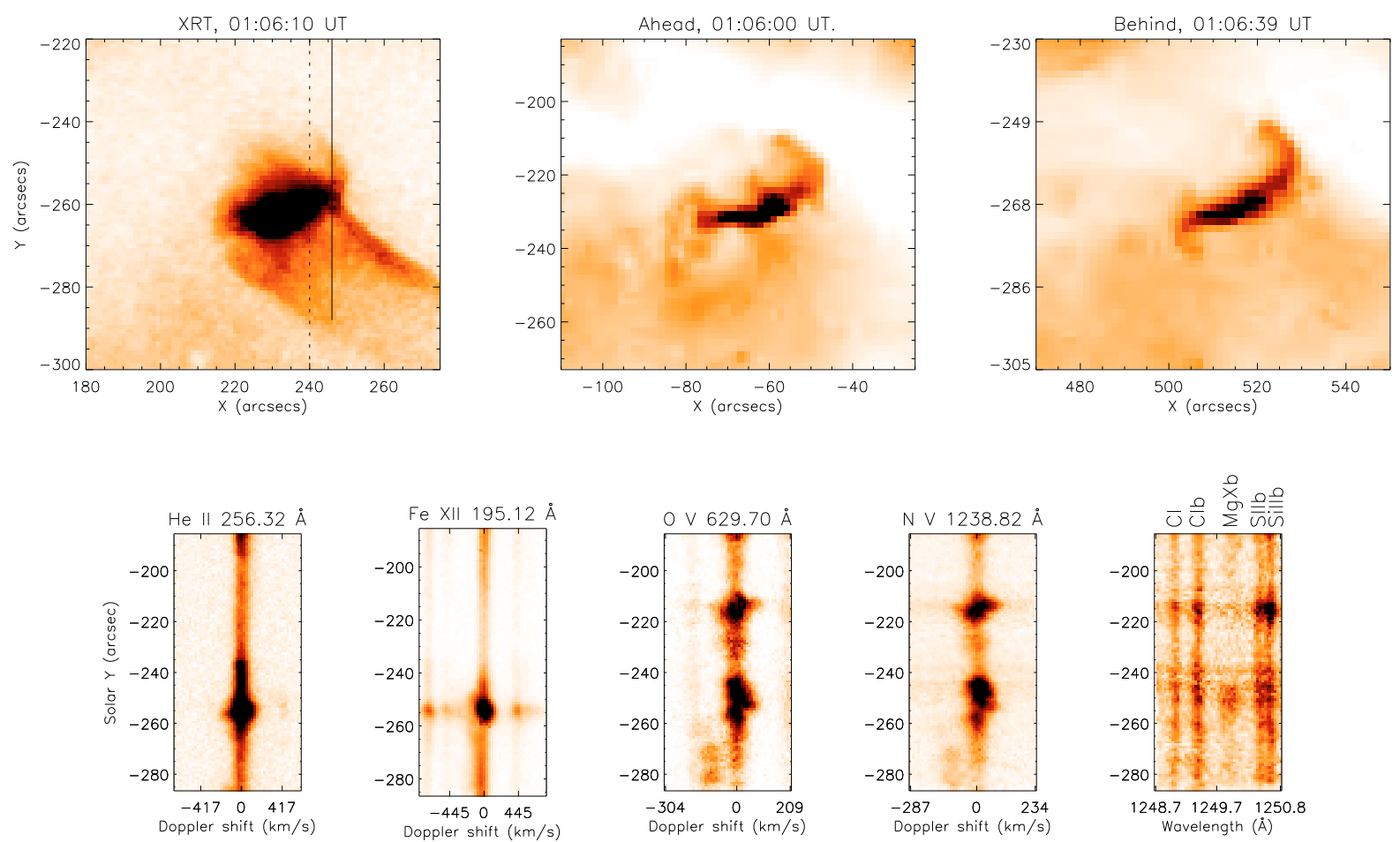

Fig. 14. The same as Fig. 11 with EIS at 01:05:50 UT and SUMER at 01:05:32 UT. 
A\&A 526, A19 (2011)
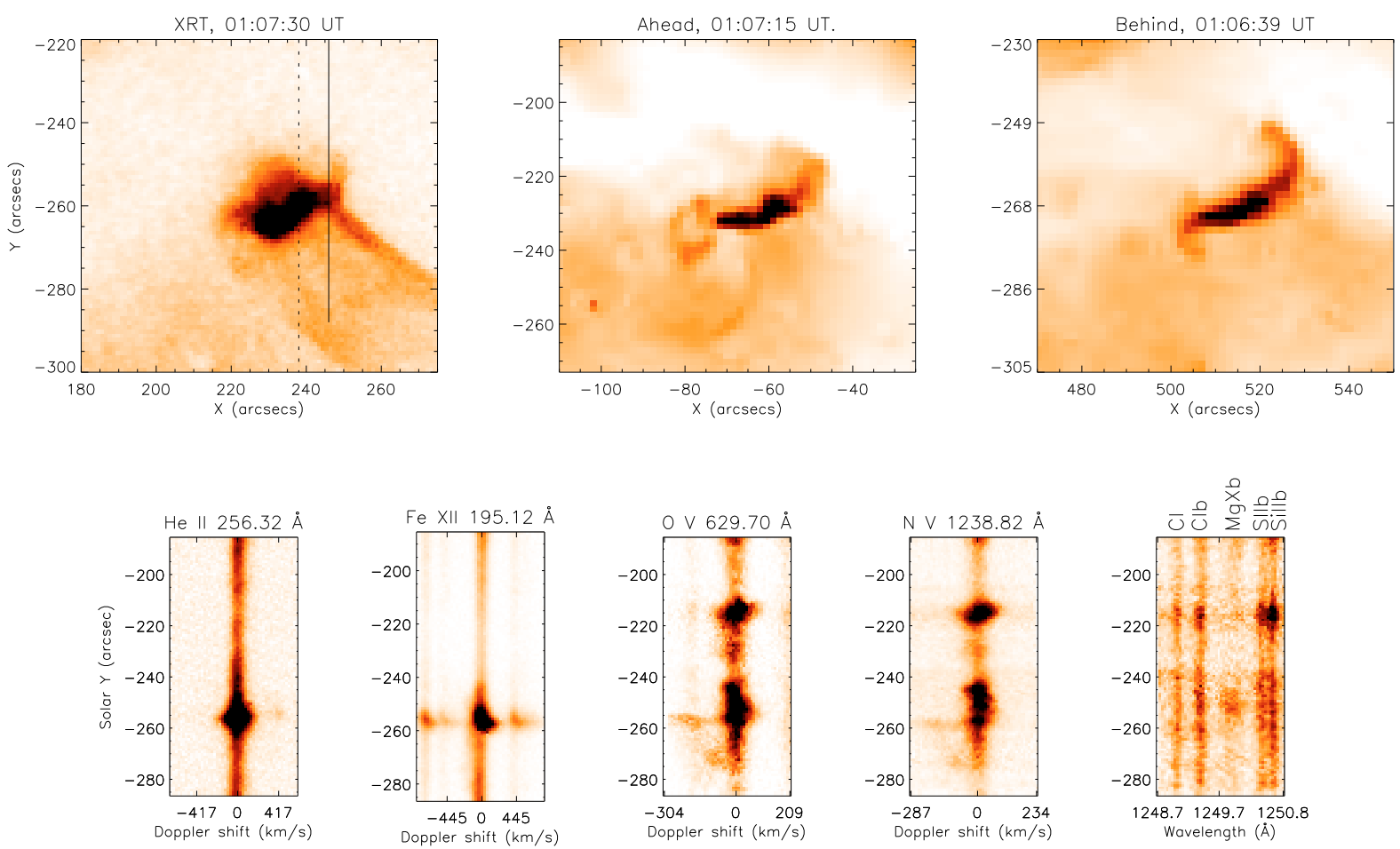

Fig. 15. The same as Fig. 11 with EIS at 01:06:52 UT and SUMER at 01:06:32 UT.
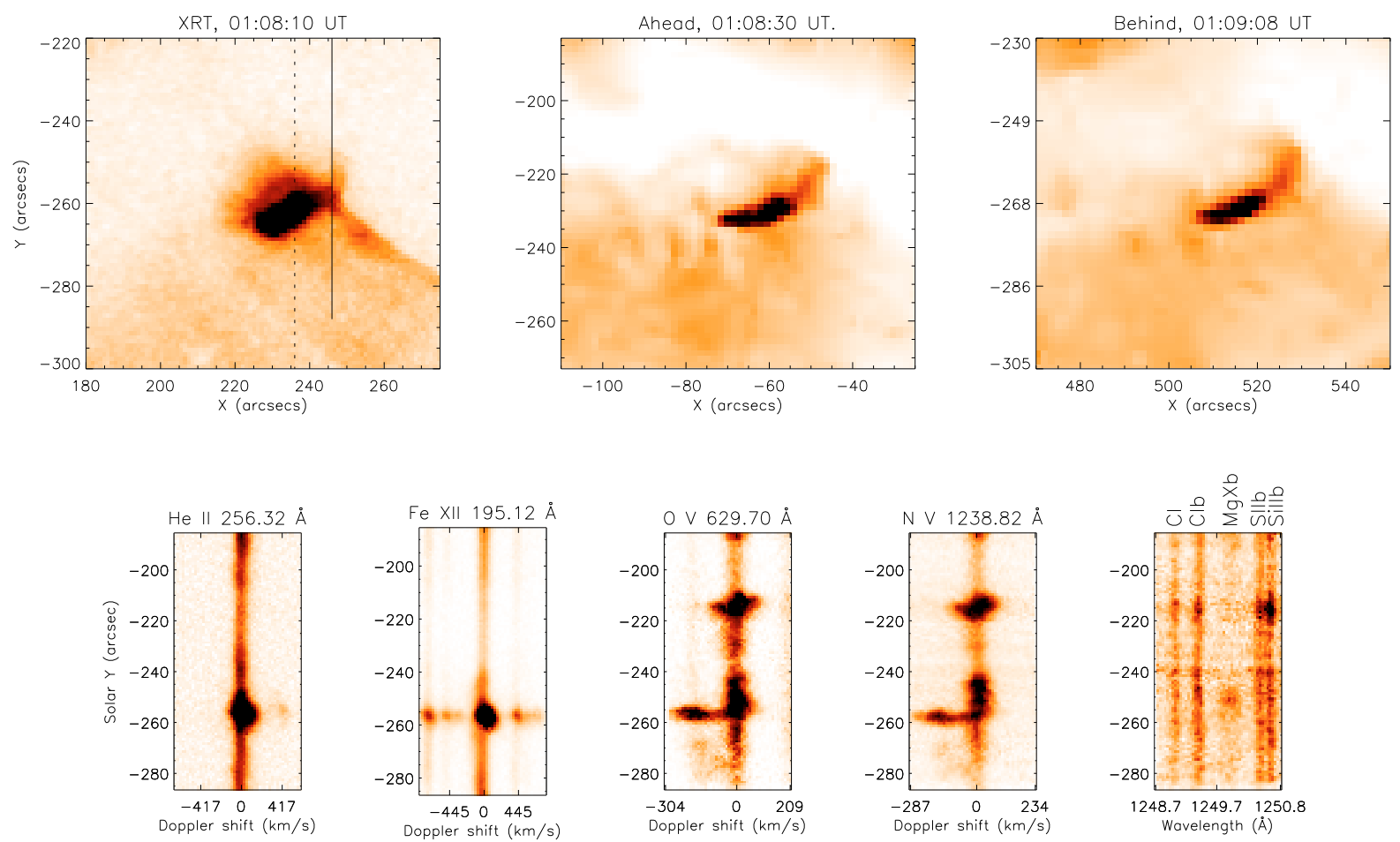

Fig. 16. The same as Fig. 11. with EIS at 01:07:54 UT and SUMER at 01:07:40 UT. 